\title{
SUPERCRITICAL INSTABILITY OF DIRAC ELECTRONS IN THE FIELD OF TWO OPPOSITELY CHARGED NUCLEI
}

\begin{abstract}
The Dirac equation for an electron in a finite dipole potential has been studied within the method of linear combination of atomic orbitals (LCAO). The Coulomb potential of the nuclei that compose a dipole is regularized, by considering the finite nuclear size. It is shown that if the dipole momentum reaches a certain critical value, the novel type of supercriticality occurs; namely, the wave function of the highest occupied electron bound state changes its localization from the negatively charged nucleus to the positively charged one. This phenomenon can be interpreted as a spontaneous creation of an electron-positron pair in vacuum, with each of the created particles being in the bound state with the corresponding nucleus and partially screening it.
\end{abstract}

Keywords: supercritical instability, wave-function localization change, critical dipole moment, LCAO method.

\section{Introduction}

The spectrum of a hydrogen-like atom has always been one of the first test that every new wave equation was undergone. The equation written down by Dirac in 1928 was not an exception [1]. In the same year, Darwin and Gordon [2,3] obtained energy levels for a one-electron atom with the nuclear charge $Z e$ :

$E=m c^{2}\left[1+\frac{(Z \alpha)^{2}}{\left(\sqrt{(j+1 / 2)^{2}-(Z \alpha)^{2}}+n_{r}\right)^{2}}\right]^{-1 / 2}$

where $j$ is the quantum number that characterizes the total angular momentum, and $n_{r}$ is the radial quantum number. For the ground state, $j=1 / 2$ and $n_{r}=$ $=0$, so that $E_{0}=m c^{2} \sqrt{1-(Z \alpha)^{2}}$. According to this formula, the ground-state energy becomes imaginary for superlarge nuclear charges, $Z>\frac{1}{\alpha} \approx 137$. This phenomenon was called the "falling to the center".

(C) O.O. SOBOL, 2016

ISSN 2071-0194. Ukr. J. Phys. 2016. Vol. 61, No. 9
In 1945, I.Ya. Pomeranchuk and Ya.A. Smorodinsky showed [4] that this falling to the center results from the singularity of the exact Coulomb potential at the coordinate origin. The solution of the relativistic Kepler problem with regard for finite dimensions of an atomic nucleus demonstrated that there are no features at $Z=137$ : the ground energy level monotonically falls down, crosses the zero, and, if the socalled critical value of nuclear charge, $Z_{\mathrm{cr}} \approx 170$, is exceeded, plunges into the lower continuum, so that the system becomes unstable with respect to the creation of electron-positron pairs $[5,6]$. The electrons fill the $K$-shell and partially screen the nucleus charge, whereas the free positrons escape to infinity. However, there are no nuclei with so large charges; therefore, the effect was not observed.

Later, there emerged an idea concerning front (or almost front) collisions between the nuclei of heavy atoms, for example, uranium [5, 7-9]. In this case, their total charge exceeds the critical value, and there exists such a distance between the nuclei, at which the lowest bound state immerses into the lower con- 
tinuum. This distance is also called critical. Unfortunately, in the relativistic problem with two centers, the variables cannot be separated in any coordinate system, so that an analytic solution cannot be constructed $[5,6]$. However, the corresponding calculations were performed with the use of approximate quantum-mechanical methods, in particular, the variational one [10], and the dependences of the critical distance between the nuclei on the total charge of the system were obtained.

Interest to the supercritical instability problem considerably grew after the experimental discovery of graphene in 2004 [11]. Really, the low-energy electron excitation in this two-dimensional crystal have an ultrarelativistic dispersion law (a Dirac cone), being described by a $(2+1)$-dimensional massless Dirac equation [12-14]. Using a series of experimental techniques, it is possible to create a mass gap in the graphene spectrum $[13,15,16]$ and obtain a $(2+1)$ dimensional analog of quantum electrodynamics. In this case, the Fermi velocity plays the role of light speed, $v_{\mathrm{F}} \approx \frac{c}{300}$, so that the coupling constant (an analog of the fine-structure constant) is much larger, $\tilde{\alpha}=\frac{e^{2}}{\hbar v_{\mathrm{F}}} \approx \frac{300}{137} \approx 2.2$. Although this value becomes appreciably smaller owing to the dielectric permittivity of the substrate for graphene, the critical impurity charge, at which the supercritical instability can be induced, remains comparable with unity [17]. Recently, the phenomenon of supercritical instability in clusters of charged $\mathrm{Ca}$ dimers was experimentally found [18]. In works $[19,20]$, the supercritical collapse in the simplest cluster composed of two identical impurities was studied, and the dependence of the critical distance between them on their charge and mass gap was calculated. In the case of gapless graphene, the instability was shown to arise, when the total charge of impurities exceeds the critical value irrespective of the distance between the impurities.

In work [21], a problem of two different impurities in graphene with a gap was considered. Confining the analysis to the point-like dipole case, the cited authors examined the features in the discrete spectrum of an electron in a vicinity of continua and revealed an exponential condensation of levels, which is similar to the Efimov scaling. However, a conclusion was drawn that there cannot be any supercritical instability in this system. This problem was also considered in works $[22,23]$. The supercritical instability was shown to take place in the case of finite dipole, but it should manifest itself differently: as a migration of the wave function of the highest filled electron state. This phenomenon was interpreted as a creation of an electron-hole pair in vacuum, with each of the created particles being bound with the corresponding impurity and screening its charge. This research stimulated the consideration of a similar problem in the $(3+1)$-dimensional QED, which is carried out in the presented work.

The non-relativistic problem with the Schrödinger equation was repeatedly considered in the literature [24-27]. It was found that, in the three-dimensional space, there is a critical value for the dipole moment, below which no bound states can exist in the system:

$D_{\mathrm{cr}}=0.6393 e a_{0}$,

where $a_{0}=\frac{\hbar^{2}}{m e^{2}}=0.529 \AA$ is the Bohr radius. In work [27], it was also shown that the corresponding critical values of dipole moment equal zero in oneand two-dimensional spaces, i.e. an arbitrarily small dipole moment would generate bound states.

The relativistic problem with the Dirac equation for an electron in the dipole potential was considered earlier in work [28]. The cited authors studied the energy behavior, as well as the corresponding wave functions, in the regions near the continua using the method of asymptotic matching. At large distances from the dipole, the squared Dirac equation has the form of Schrödinger equation and allows an asymptotic separation of variables, which makes it possible to determine the critical dipole moment. The latter is approximately equal to non-relativistic value (2).

The problem with the electric dipole is evidently symmetric with respect to the charge inversion. This symmetry plays the crucial role and gives rise to a symmetric arrangement of energy levels relatively to zero. As a result, the electron and positron (symmetric to it) states could have intersected only at $E=0$. However, this is impossible, because those states have identical quantum numbers, and the Wignervon Neumann theorem on the absence of a crossing of levels is applicable [29]. This circumstance brings about the existence of characteristic "constrictions" in the spectrum (cf. Figs. 5 and 7 below).

The consideration of the Dirac equation with an electric dipole field has also a relation to the description of the heavy meson decay [6]. Really, a heavy quark and an antiquark have opposite color charges 
and, therefore, form a dipole. In the color field of this dipole (which, owing to the confinement phenomenon, increases when the quarks are attempted to move some distance apart), a pair of a light quark and an antiquark can be created. This pair gets bound with a heavy quark and an antiquark and screens their charges. As a result, the decay of one heavy meson gives rise to the emergence of two lighter ones.

In this work, the method of linear combination of atomic orbitals (LCAO) will be applied to calculate the spectrum and the wave functions of an electron in the potential created by a finite electric dipole. The Coulomb potential of the nuclei that form the dipole was regularized, by taking their finite dimensions into account. The wave functions of several first electron levels in the field of one nucleus, which are centered at the corresponding nucleus, were used as atomic orbitals. Attempts were made to extend the limits of applicability of the LCAO method to supercritical nuclear charges. The corresponding calculations were carried out not only near the edges of continua, but over the whole mass gap, which has not been studied earlier in the literature.

The paper is organized as follows. In Section 2, an exact solution of the problem with a regularized potential of one nucleus is quoted, and various types of regularizations are analyzed. In Section 3, within the LCAO method, the energy and wave functions of an electron in the dipole potential are calculated, and their behavior depending on the dipole moment variation is considered. Section 4 is devoted to the extension of the limits of applicability of the LCAO method to nuclei with large charge values. The obtained results are generalized and the conclusions are drawn in Section 5. Some technical calculations that arise in the course of the LCAO method application are presented in Appendix.

\section{Regularized Potential of a Single Nucleus}

Let us consider the motion of an electron in the potential of a charged nucleus. The corresponding Hamiltonian looks like

$\hat{H}=-i \hbar c \gamma^{0} \gamma \nabla+\gamma^{0} m c^{2}-Z e^{2} v(r)$,

where the function $v(r)$ describes the regularized Coulomb potential, and $\gamma^{\mu}(\mu=0, \ldots, 3)$ are the Dirac matrices, for which the standard Dirac-Pauli representation is used.

ISSN 2071-0194. Ukr. J. Phys. 2016. Vol. 61, No. 9
To make calculations convenient, let us change to dimensionless variables. In what follows, all energy quantities will be measured in terms of electron rest mass units, $E_{0}=m c^{2} \approx 0.511 \mathrm{MeV}$, making no changes of their notations. All distances will be measured in terms of the Compton wavelength for the electron, $\lambda_{C}=\frac{\hbar}{m c} \approx 386 \mathrm{fm}$. We also introduce the notation $\zeta=Z \alpha$, where $\alpha=\frac{e^{2}}{\hbar c} \approx \frac{1}{137}$ is the finestructure constant.

The potential of a charged nucleus is spherically symmetric, so that the total angular momentum remains constant. The wave functions, which are characteristic of the operators $\hat{J}^{2}$ and $\hat{J}_{z}$, should be sought in the form

$|\Psi\rangle=\left(\begin{array}{c}g(r) \Omega_{j, j_{z}, l} \\ i f(r) \Omega_{j, j_{z}, l^{\prime}}^{\prime}\end{array}\right)$.

Here, $l=j-1 / 2, l^{\prime}=l+1=2 j-l$, the wave function parity equals $(-1)^{l}$, and the angular dependences are described by the spherical spinors $\Omega$ and $\Omega^{\prime}$, which look like

$$
\begin{aligned}
\Omega_{j, j_{z}, l} & =\left(\begin{array}{l}
\sqrt{\frac{j+j_{z}}{2 j}} Y_{l, j_{z}}-1 / 2 \\
\sqrt{\frac{j-j_{z}}{2 j}} Y_{l, j_{z}+1 / 2}
\end{array}\right), \\
\Omega_{j, j_{z}, l^{\prime}}^{\prime} & =\left(\begin{array}{c}
-\sqrt{\frac{j-j_{z}+1}{2 j+2}} Y_{l^{\prime}, j_{z}-1 / 2} \\
\sqrt{\frac{j+j_{z}+1}{2 j+2}} Y_{l^{\prime}, j_{z}+1 / 2}
\end{array}\right),
\end{aligned}
$$

where the spherical harmonics are designated in the standard way [30],

$$
\begin{aligned}
& Y_{l, m}(\theta, \varphi)=(-1)^{\frac{m+|m|}{2}} i^{l} \sqrt{\frac{2 l+1}{4 \pi} \frac{(l-|m|) !}{(l+|m|) !}} \times \\
& \times P_{l}^{|m|}(\cos \theta) e^{i m \varphi} .
\end{aligned}
$$

The wave functions that have, for the same $j$-value, the opposite parity in comparison with the functions indicated in Eq. (4) are tried in a similar form, in which the spherical spinors $\Omega$ and $\Omega^{\prime}$ should be swapped,

$|\tilde{\Psi}\rangle=\left(\begin{array}{c}i \tilde{g}(r) \Omega_{j, j_{z}, l^{\prime}}^{\prime} \\ -\tilde{f}(r) \Omega_{j, j_{z}, l}\end{array}\right)$,

where the additional phase multiplier $i$ was introduced for the sake of convenience. 
After substituting expressions (4) and (7) into the Dirac equation $\hat{H}|\Psi\rangle=E|\Psi\rangle$, the following systems of ordinary differential equations for the radial functions are obtained:

$$
\begin{aligned}
& \left\{\begin{array}{l}
g^{\prime}-\frac{j-1 / 2}{r} g-f(1+E+\zeta v(r))=0, \\
f^{\prime}+\frac{j+3 / 2}{r} f-g(1-E-\zeta v(r))=0,
\end{array}\right. \\
& \left\{\begin{array}{l}
\tilde{g}^{\prime}+\frac{j+3 / 2}{r} \tilde{g}-\tilde{f}(1+E+\zeta v(r))=0, \\
\tilde{f}^{\prime}-\frac{j-1 / 2}{r} \tilde{f}-\tilde{g}(1-E-\zeta v(r))=0 .
\end{array}\right.
\end{aligned}
$$

The normalization condition for the wave functions, which involves the orthonormal character of spherical spinors, acquires the form

$$
\begin{aligned}
\langle\Psi \mid \Psi\rangle & =\int_{0}^{+\infty} r^{2}\left(f^{2}(r)+g^{2}(r)\right) d r=1, \\
\langle\tilde{\Psi} \mid \tilde{\Psi}\rangle & =\int_{0}^{+\infty} r^{2}\left(\tilde{f}^{2}(r)+\tilde{g}^{2}(r)\right) d r=1 .
\end{aligned}
$$

For every fixed value of quantum number $j$, the systems of equations (8) and (9) have infinite numbers of solutions that satisfy the normalization conditions (10). Therefore, let us mark those solutions by the radial quantum number $n_{r}=0,1,2, \ldots$. Furthermore, let us designate the orbital quantum number $L$ of the spherical spinor that appears in the upper component of the wave function by capital Latin letters following the conventional order: $S(L=0), P(L=1)$, $D(L=2), F(L=3)$, and so on. By analogy with the non-relativistic case, let us also introduce the principal quantum number $n=n_{r}+L+1$. The RussellSaunders notation [31] will be used to designate terms, and, additionally, the projection of the angular momentum will be indicated: $\left|n L_{j}, j_{z}\right\rangle$. Hence, the first (by energy) three states have the following sets of quantum numbers:

1) $j=1 / 2, j_{z}= \pm 1 / 2, n_{r}=0, L=l=0, l^{\prime}=1$,

$n=1 \rightarrow\left|1 S_{1 / 2}, \pm 1 / 2\right\rangle$;

2) $j=1 / 2, j_{z}= \pm 1 / 2, n_{r}=0, L=l^{\prime}=1, l=0$,

$n=2 \rightarrow\left|2 P_{1 / 2}, \pm 1 / 2\right\rangle$;

3) $j=1 / 2, j_{z}= \pm 1 / 2, n_{r}=1, L=l=0, l^{\prime}=1$,

$n=2 \rightarrow\left|2 S_{1 / 2}, \pm 1 / 2\right\rangle$.

The problem with the regularized Coulomb potential has no analytic solution. Therefore, the systems of equations (8) and (9) have to be integrated numerically. For this purpose, we should determine boundary conditions for the radial functions at $r=0$. Let us express $f$ (or $\tilde{f}$ ) from the first equation of the system and substitute it into the second one. Let the characteristic regularization scale be equal to $r_{0}$. Then, if $r<r_{0}$, we obtain $v(r) \approx v(0) \gg 1$. The asymptotics can be determined from the approximate equation

$g^{\prime \prime}+\frac{2}{r} g^{\prime}+\left(\zeta^{2} v^{2}(0)-\frac{j^{2}-1 / 4}{r^{2}}\right) g=0$,

Its solution that is regular at $r=0$ looks like

$g(r) \sim \frac{1}{\sqrt{r}} J_{j}(\zeta v(0) r)$.

The asymptotic behavior of the solutions of system (9) is analyzed analogously. At $j=1 / 2$, the boundary conditions can be chosen in the form

$$
\begin{aligned}
& g(0)=1, \quad f(0)=0 \\
& \tilde{g}(0)=0, \quad \tilde{f}(0)=1 .
\end{aligned}
$$

The spectrum is determined from the condition that the wave function have to fall down exponentially as $r \rightarrow \infty$.

The result depends on the specific manner of the potential regularization. The following three variants should be considered:

(i) regularization by "going into the fourth dimension":

$v_{I}\left(r, r_{0}\right)=\frac{1}{\sqrt{r^{2}+r_{0}^{2}}} ;$

(ii) regularization by considering the finite nuclear size (the nuclear charge is uniformly distributed over the nuclear surface):

$v_{\mathrm{II}}\left(r, r_{0}\right)= \begin{cases}\frac{1}{r}, & r \geq r_{0} \\ \frac{1}{r_{0}}, & r<r_{0} .\end{cases}$

(iii) regularization by considering the finite nuclear size (the nuclear charge is uniformly distributed over the nuclear volume):

$v_{\mathrm{III}}\left(r, r_{0}\right)= \begin{cases}\frac{1}{r}, & r \geq r_{0} ; \\ \frac{1}{r_{0}} \frac{3-\left(r / r_{0}\right)^{2}}{2}, & r<r_{0} .\end{cases}$

ISSN 2071-0194. Ukr. J. Phys. 2016. Vol. 61, No. 9 
To model the real value of regularizing parameter $r_{0}$, let us use the empirical relation for the nuclear radius, which is known in nuclear physics [32]:

$r_{0} \approx 1.25 \mathrm{fm} \cdot A^{1 / 3}$,

where $A=Z+N$ is the nuclear mass number. In order to find the dependence of nuclear radius on the nuclear charge, let us adopt that the nucleus lies in the so-called beta-stability valley, for which the following approximate relation is obeyed [32]:

$Z=\frac{A}{1.98+0.015 A^{2 / 3}}$.

By solving the cubic equation, we obtain the dependence $A=A(Z)$. Afterward, from Eq. (18), we calculate the dependence $r_{0}=r_{0}(Z)$. The latter is shown in Fig. 1.

Figure 2 demonstrates the dependences of the ground-state energy of the electron in the field of nucleus on the nuclear charge for three kinds of regularization. For comparison, a similar curve for the case of non-regularized potential $E_{N R}=\sqrt{1-(Z \alpha)^{2}}$ is also depicted. From the presented curves, one can see that the "falling to center" phenomenon takes place for the non-regularized potential, if $Z \gtrsim 137$, i.e. when the ground-state energy becomes imaginary, and the system has no definite ground state. This problem is eliminated, if the potential is regularized in any fashion. The ground-state energy monotonically falls down and becomes negative at a certain threshold charge value. Depending on the regularization type, the threshold charge value falls within the interval $148 \leq Z_{0} \leq 152$. Then the energy continues to decrease monotonically and, when achieving the critical value, the level plunges into the lower continuum, inducing the supercritical instability. Depending on the regularization type, the critical charge falls within the interval $170 \leq Z_{\text {cr }} \leq 186$.

The most natural is the regularization of the third kind (i.e. a uniform charge distribution in a nucleus with finite dimensions). Therefore, it will be used below in our all numerical calculations, although, for the sake of generality, an arbitrarily regularized potential $v(r)$ will be retained in all relevant formulas.

In Fig. 3, the energy dependences on the absolute value of nuclear charge are exhibited for three first levels (11) of an electron in the regularized Coulomb potential (regularization of type iii). The levels start

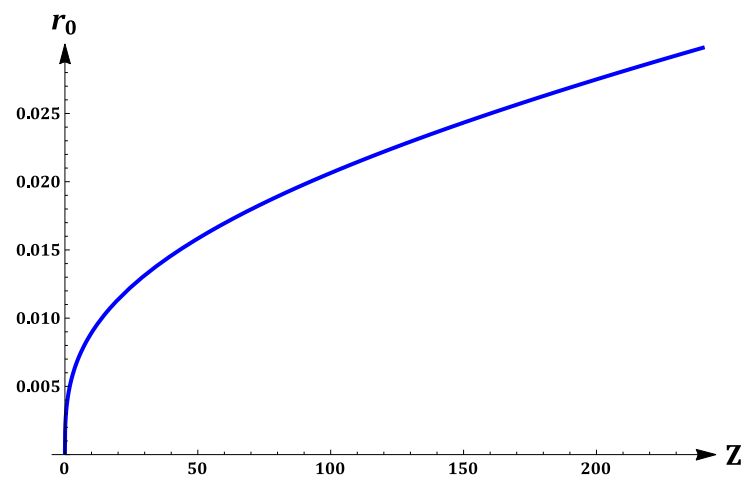

Fig. 1. Dependence of the regularizing parameter $r_{0}$ on the nuclear charge $Z$

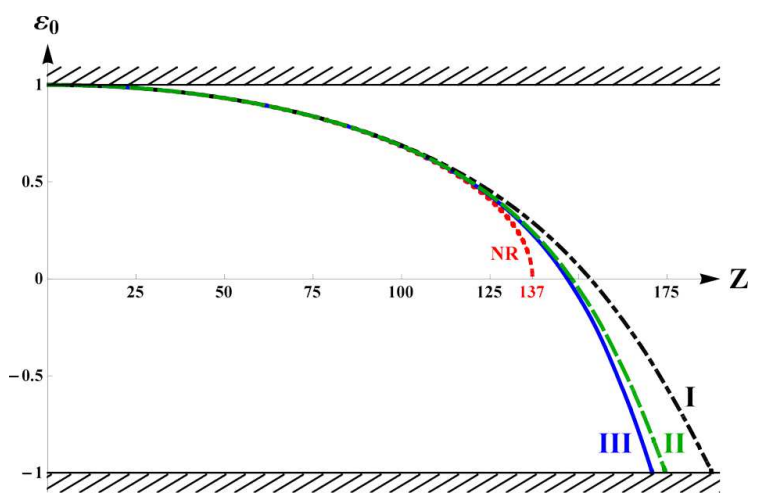

Fig. 2. Dependences of the ground-state energy of an electron in the regularized Coulomb potential on the nuclear charge for various regularization models: I (dash-dotted curve), II (dashed curve), and III (solid curve). For comparison, the same dependence for the non-regularized (NR) potential is shown (dotted curve)

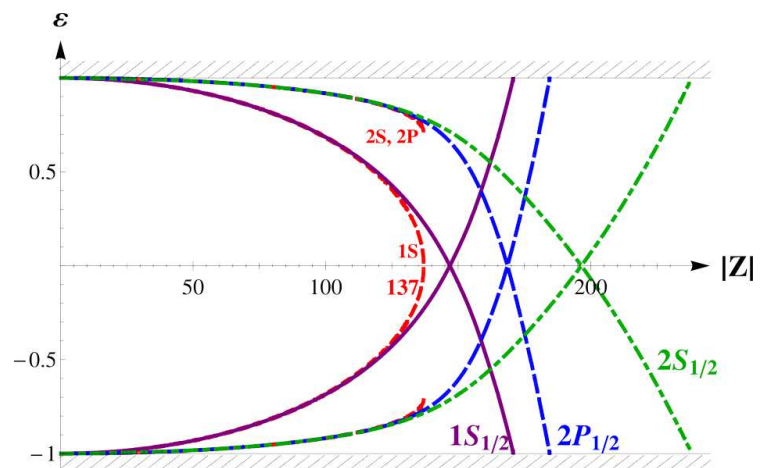

Fig. 3. Energy dependences on the nuclear charge for three lowest electron levels (11) in the regularized Coulomb potential: $1 S_{1 / 2}$ (solid curves), $2 P_{1 / 2}$ (dashed curves), and $2 S_{1 / 2}$ (dash-dotted curves). Red dashed curves exhibit the energy of corresponding levels in the non-regularized Coulomb potential 


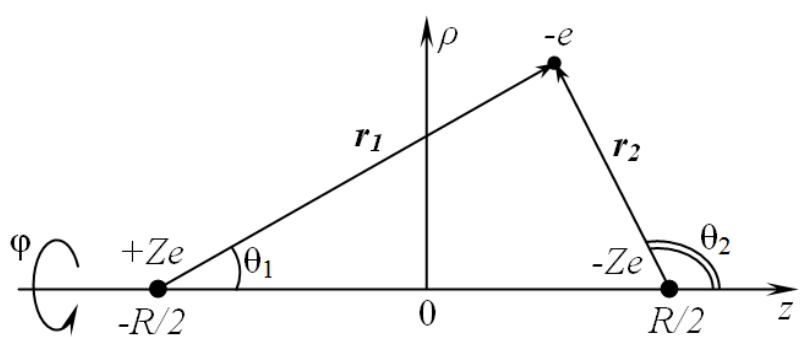

Fig. 4. Schematic diagram of an electron in the field of an electric dipole

from the upper continuum for a positively charged nucleus and, symmetrically, from the lower continuum for a negatively charged one. On the basis of those three energy levels, the LCAO method for finding the energy and wave functions of an electron in the dipole potential will be developed in the next sections.

\section{LCAO Method \\ for the Problem with a Dipole}

Now let us consider an electron in the potential of a finite electric dipole. The corresponding dimensionless Hamiltonian looks like:

$\hat{H}=-i \gamma^{0} \gamma \nabla+\gamma^{0}-\zeta\left(v\left(r_{1}\right)-v\left(r_{2}\right)\right)$,

where $r_{1,2}=\sqrt{(z \pm R / 2)^{2}+\rho^{2}}$ and $\rho=\sqrt{x^{2}+y^{2}}$ (see Fig. 4). Unfortunately, none of coordinate systems allow the variables in this problem to be separated. Therefore, approximate methods will be applied. The arrangement of the system in the space, coordinate system, and some notations are shown in Fig. 4.

Let us perform the analysis, by using the LCAO method. This technique is well-known and widely applied in molecular physics [33]. In this method, the wave functions are selected as linear combinations of basis functions. The latter, as a rule, are one-electron functions localized at the corresponding atoms in the molecule concerned. The coefficients in a linear combination are determined by minimizing the total energy of the system.

The Hamiltonians for an electron in the fields of positively and negatively charged nuclei will be denoted as, respectively,

$\hat{H}_{ \pm}(\zeta)=-i \gamma^{0} \gamma \nabla+\gamma^{0} \pm \zeta v(r)$.
The charge conjugation operator

$\hat{U}_{c}=-i \gamma^{2} \hat{K}$

where $K$ is the complex conjugation operator, and $\hat{U}_{c}^{2}=1$, transforms those Hamiltonians into each other:

$\hat{U}_{c} \hat{H}_{+}(\zeta) \hat{U}_{c}=-\hat{H}_{-}(\zeta)$.

Therefore, if $|\Psi\rangle$ is a characteristic function for the Hamiltonian $H_{-}(\zeta)$ with energy $E$, the function $\left|\Psi_{c}\right\rangle=\hat{U}_{c}|\Psi\rangle$ is a characteristic function for the Hamiltonian $\hat{H}_{+}(\zeta)$ with the energy $-E$.

Using the explicit expressions for the spherical spinors, it is easy to get convinced that

$$
\begin{aligned}
& \sigma_{2} \Omega_{j, j_{z}, l}^{*}=-i(-1)^{(l+1)-j_{z}+1 / 2} \Omega_{j,-j_{z}, l}, \\
& \sigma_{2} \Omega_{j, j_{z}, l^{\prime}}^{\prime *}=-i(-1)^{l^{\prime}-j_{z}+1 / 2} \Omega_{j,-j_{z}, l^{\prime}}^{\prime}
\end{aligned}
$$

i.e. the charge conjugation operator changes the sign of the angular momentum projection.

The problem with the dipole potential is no more spherically symmetric. Therefore, the total momentum is not conserved. However, the axial symmetry with respect to the axis $O z$ that passes through both charged centers still exists, so that the projection of the total momentum on this axis is constant. Let us choose a test function for the LCAO method as a linear combination of wave functions with identical values of the angular momentum projection, $j_{z}=+1 / 2$ :

$|\Psi\rangle=c_{1}|1\rangle+c_{2}|2\rangle$,

where the basis states are

$|1\rangle=\left|1 S_{1 / 2},+1 / 2, r_{1}, \zeta\right\rangle=$

$=\left(\begin{array}{c}g_{0}\left(r_{1}\right) \Omega_{1 / 2,1 / 2,0}(1) \\ i f_{0}\left(r_{1}\right) \Omega_{1 / 2,1 / 2,1}^{\prime}(1)\end{array}\right)$,

$|2\rangle=\hat{U}_{c}\left|1 S_{1 / 2},-1 / 2, r_{2}, \zeta\right\rangle=$

$=\left(\begin{array}{c}i f_{0}\left(r_{2}\right) \Omega_{1 / 2,1 / 2,1}^{\prime}(2) \\ g_{0}\left(r_{2}\right) \Omega_{1 / 2,1 / 2,0}(2)\end{array}\right)$.

In these expressions, the radial functions $f_{0}(r)$ and $g_{0}(r)$ are determined by numerically integrating the

ISSN 2071-0194. Ukr. J. Phys. 2016. Vol. 61, No. 9

\section{4}


problem with one nucleus (see Section 2). The spherical spinors look like

$$
\begin{aligned}
& \Omega_{1 / 2,1 / 2,0}=\frac{1}{\sqrt{4 \pi}}\left(\begin{array}{l}
1 \\
0
\end{array}\right), \\
& \Omega_{1 / 2,1 / 2,1}^{\prime}=\frac{i}{\sqrt{4 \pi}}\left(\begin{array}{c}
\cos \theta \\
-\sin \theta e^{i \varphi}
\end{array}\right) .
\end{aligned}
$$

Let us project the Dirac equation $\hat{H}|\Psi\rangle=E|\Psi\rangle$ on each of the basis states:

$$
\begin{aligned}
& c_{1} H_{11}+c_{2} H_{12}=E\left(c_{1}+c_{2} S\right), \\
& c_{1} H_{21}+c_{2} H_{22}=E\left(c_{1} S+c_{2}\right) .
\end{aligned}
$$

Then we obtain the following secular equation for the non-trivial coefficients in the linear combination (25):

$\operatorname{det}\left|\begin{array}{cc}H_{11}-E & H_{12}-S E \\ H_{21}-S^{*} E & H_{22}-E\end{array}\right|=0$,

where $H_{i j}=\langle i|\hat{H}| j\rangle$ are the matrix elements of the Hamiltonian, and $S=\langle 1 \mid 2\rangle$ is the overlap integral, which equals zero as is shown by formula (A2) in Appendix.

In order to calculate the matrix elements, we take the total Hamiltonian in the form

$\hat{H}=\hat{H}_{-}(1)+\zeta v\left(r_{2}\right)=\hat{H}_{+}(2)-\zeta v\left(r_{1}\right)$.

Then,

$H_{11}=-H_{22}=\varepsilon_{0}+\zeta C$,

$H_{12}=H_{21}=-\zeta A$,

where $\varepsilon_{0}=\varepsilon^{(1 S)}(\zeta)$ is the ground-state energy of an electron in the field of one nucleus (see Figs. 2 and 3). The expressions for the matrix elements include the Coulomb, $C$, and exchange, $A$, integrals. Expressions for them can be found in Appendix: formulas (A19) and (A22), respectively. These integrals are calculated provided that the functions $f_{0}$ and $g_{0}$ are given.

Ultimately, we obtain a spectrum

$$
\varepsilon= \pm \sqrt{\left(H_{11}\right)^{2}+\left(H_{12}\right)^{2}}= \pm \sqrt{\left(\varepsilon_{0}+\zeta C\right)^{2}+\zeta^{2} A^{2}}
$$

which is symmetric with respect to zero and reflects the charge symmetry of the problem. For the negative

ISSN 2071-0194. Ukr. J. Phys. 2016. Vol. 61, No. 9 spectral branch, the coefficients in the linear combination (25) are as follows:

$$
\begin{gathered}
c_{1}=\frac{H_{12}}{\sqrt{H_{12}^{2}+\left(H_{11}+\sqrt{H_{11}^{2}+H_{12}^{2}}\right)^{2}}}, \\
c_{2}=-\frac{H_{11}+\sqrt{H_{11}^{2}+H_{12}^{2}}}{\sqrt{H_{12}^{2}+\left(H_{11}+\sqrt{H_{11}^{2}+H_{12}^{2}}\right)^{2}}} .
\end{gathered}
$$

It is easy to see that, as $R \rightarrow \infty$, we have $C \approx \frac{1}{R} \rightarrow 0$ and $A \sim \exp \left(-\sqrt{1-\varepsilon_{0}^{2}} R\right) \rightarrow 0$. Therefore, at large distances between the nuclei, the energy of the system $\varepsilon \rightarrow \pm\left|\varepsilon_{0}\right|$. This is quite expectedly, since we may consider the nuclei to be isolated in this case.

From expression (32) for the energy and from the fact that $C \geq 0$, one can see that the spectral "constriction" can arise only, if $\varepsilon_{0}<0$, with the minimum itself being located near the point, at which $\zeta C=\left|\varepsilon_{0}\right|$. At the same point, we have $\left|c_{1}\right|=\left|c_{2}\right|=1 / \sqrt{2}$, i.e. the wave function changes its localization:

$$
\begin{aligned}
& \left|c_{2}\right|^{2}-\left|c_{1}\right|^{2}=\frac{2 H_{11}\left(H_{11}+\sqrt{H_{11}^{2}+H_{12}^{2}}\right)}{H_{12}^{2}+\left(H_{11}+\sqrt{H_{11}^{2}+H_{12}^{2}}\right)^{2}} \sim \\
& \sim \operatorname{sign}\left(H_{11}\right) .
\end{aligned}
$$

The quantities $C$ and $|A|$ monotonically decrease with the growth of the distance $R$. Initially, $\left|c_{2}\right|>\left|c_{1}\right|$, i.e. the electron charge density is mainly localized at the negatively charged nucleus. After passing the "constriction", we obtain $\left|c_{2}\right|<\left|c_{1}\right|$, i.e. the wave function changes its localization to the positively charged nucleus.

One can also see that if $\varepsilon_{0}>0$, we always obtain $H_{11}>0$ and $\left|c_{2}\right|>\left|c_{1}\right|$. As a result, the wave function remains mainly localized at the negatively charged nucleus, i.e. its localization does not change.

Figure 5 exhibits energy curves for the ground state of an electron in the field of an electric dipole that were calculated for two values of nuclear charge. At $\zeta=0.95(Z=130)$, the ground-state energy of an electron in the field of one nucleus is positive $\left(\varepsilon_{0}=+0.35\right.$, see Fig. 3$)$; therefore, the electron energy monotonically changes in the dipole field as the dipole moment grows (dashed curves in Fig. 5). At $\zeta=1.14(Z=156)$, the ground state of an electron in the field of one nucleus is negative $\left(\varepsilon_{0}=-0.30\right.$, see Fig. 3); therefore, the electron energy in the dipole field passes a "constriction" (solid curves in Fig. 5), and the wave function of the highest filled 


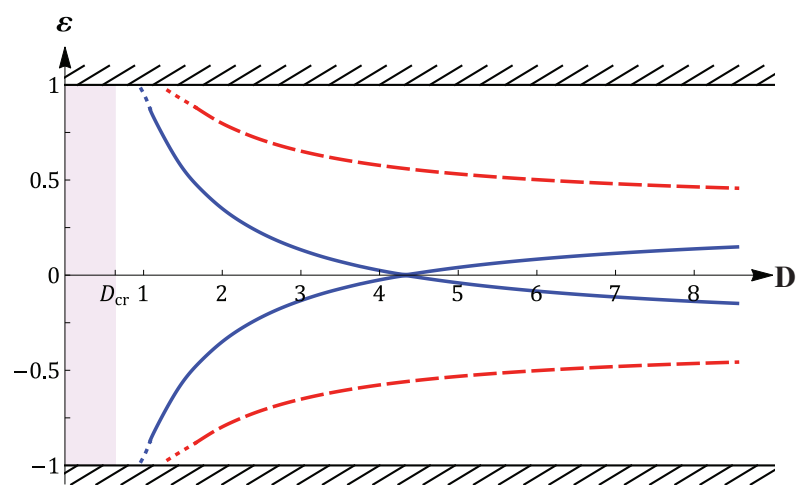

Fig. 5. Dependences of the ground-state energy of an electron in the field of an electric dipole on the dimensionless dipole moment $D=\frac{D_{\mathrm{ph}}}{e a_{0}}=\zeta R$ for two values of nuclear charge: $Z=130$ (dashed curves) and 156 (solid curves). The tinted area marks the region with no bound states [see formula (2)]

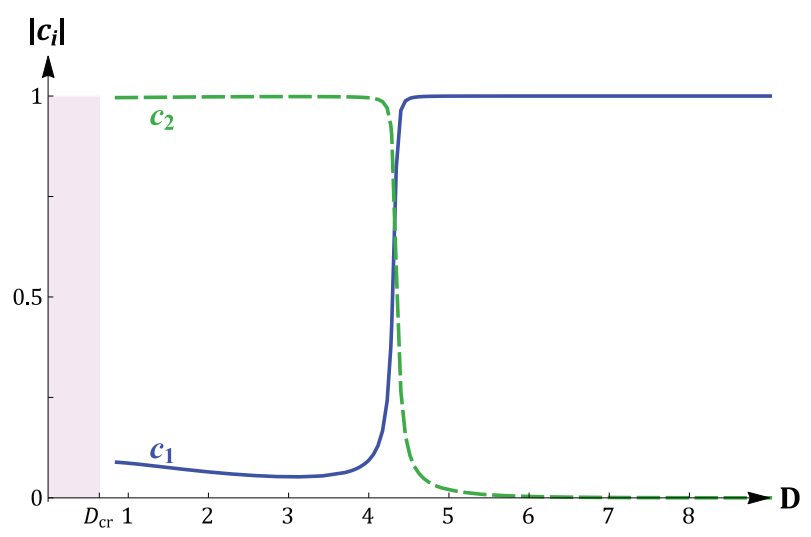

Fig. 6. Dependences of the coefficients in the linear combination (25) for the highest filled electron state (the lower solid curve in Fig. 5) on the dimensionless dipole moment $D=\frac{D_{\mathrm{ph}}}{e a_{0}}=\zeta R$ for the nuclear charge $Z=156$. The phenomenon of wave-function localization change can be observed

state changes its localization at this moment from the negatively charged nucleus to the positively charged one (Fig. 6).

All levels with negative energies start from the lower continuum, when the dipole moment exceeds some critical value (2). All of them are filled with electrons from the Dirac sea. Those "sea" electrons are initially localized at the negatively charged nucleus. Then, as the distance between the nuclei increases (the dipole moment grows), the wave function of one of those sea electrons migrates to the positively charged nucleus. A hole (positron) remains at its place near the negatively charged nucleus. In such a manner, we obtain the electron-positron pair created from vacuum. Each of the particles is in the bound state with the corresponding nucleus and partially screens its charge. It should be noted that, for this phenomenon to emerge, the nuclear charges must be so large that the level of an electron in the field of a positive nucleus would cross the energy zero, or the level of an electron in the field of a positive nucleus and its symmetric counterpart in the field of a negative nucleus would together overcome the energy gap equal to $2 m c^{2}$.

The described phenomenon is very similar to the supercritical atomic collapse. The difference consists in that, in the collapse case, we have one nucleus with the charge $Z \gtrsim 170$, and, in its field, the lowest electron bound state can reach the lower continuum on its own (in so doing, it crosses the energy distance $2 m c^{2}$ ). The system becomes unstable with respect to the creation of electron-positron pairs. The created electrons are localized at the nucleus and partially screen its charge, whereas the free positrons escape to infinity.

In the framework of this consideration, a shortcoming of the LCAO method is the fact that the asymptotic behavior of the wave functions at infinity is $\sim e^{-\sqrt{1-\varepsilon_{0}^{2}} r}$ rather than $\sim e^{-\sqrt{1-\varepsilon^{2}} r}$, as it should be. This shortcoming is especially pronounced at short distances between the nuclei, $R$, when the true energy of the system tends to the continuum boundaries, so that the exponential damping has to be weak. In this method, however, the wave functions are constructed from the wave functions obtained in the problem with one center. Therefore, the character of their damping is identical at any distance, being only determined by the ground state energy of an electron in the field of one center. Hence, the method concerned is inapplicable at small $R$.

The described method is based only on two terms in the linear combination (25). In other words, it takes into account only the ground state of an electron in the field of one nucleus. This scenario is rather good for relatively small charges. However, if the charge of nuclei is so large that the ground level in the field of a positive nucleus and the second level in the field of a negative nucleus have approximately the same energy $(Z \gtrsim 160$, see Fig. 3 ), the linear combination has also to include the wave functions of the second energy level. The situation becomes even more worsened for supercritical values of nuclear charges, when 
the ground level plunges into the lower continuum ( $Z \gtrsim 170$, see Fig. 3 ). In this case, the ground-state energy becomes complex, and the wave functions nonnormalized, so that the application of the latter as basis functions becomes impossible. These difficulties can be resolved by making a certain modification to the LCAO method, which is described in the next section.

\section{Extension of Applicability Range of LCAO Method}

In order to overcome the difficulties described above, let us try the wave function as the following linear combination (the indicated number of terms is enough for nuclei with the charges of nuclei $Z \lesssim 185$; for larger charges, the next excited states should be taken into consideration):

$|\Psi\rangle=\sum_{i=1}^{6} c_{i}|i\rangle$,

where the states $|i\rangle$ look like:

$|1\rangle=\left|1 S_{1 / 2},+1 / 2, r_{1}, \zeta_{a}\right\rangle=$

$=\left(\begin{array}{c}g_{0}\left(r_{1}\right) \Omega_{1 / 2,1 / 2,0}(1) \\ i f_{0}\left(r_{1}\right) \Omega_{1 / 2,1 / 2,1}^{\prime}(1)\end{array}\right)$,

$|2\rangle=\hat{U}_{c}\left|1 S_{1 / 2},-1 / 2, r_{2}, \zeta_{a}\right\rangle=$

$=\left(\begin{array}{c}i f_{0}\left(r_{2}\right) \Omega_{1 / 2,1 / 2,1}^{\prime}(2) \\ g_{0}\left(r_{2}\right) \Omega_{1 / 2,1 / 2,0}(2)\end{array}\right)$,

$|3\rangle=\left|2 P_{1 / 2},+1 / 2, r_{1}, \zeta_{b}\right\rangle=$

$=\left(\begin{array}{c}i \tilde{g}_{0}\left(r_{1}\right) \Omega_{1 / 2,1 / 2,1}^{\prime}(1) \\ -\tilde{f}_{0}\left(r_{1}\right) \Omega_{1 / 2,1 / 2,0}(1)\end{array}\right)$,

$|4\rangle=\hat{U}_{c}\left|2 P_{1 / 2},-1 / 2, r_{2}, \zeta_{b}\right\rangle=$

$=\left(\begin{array}{c}\tilde{f}_{0}\left(r_{2}\right) \Omega_{1 / 2,1 / 2,0}(2) \\ -i \tilde{g}_{0}\left(r_{2}\right) \Omega_{1 / 2,1 / 2,1}^{\prime}(2)\end{array}\right)$,

$|5\rangle=\left|2 S_{1 / 2},+1 / 2, r_{1}, \zeta\right\rangle=$

$=\left(\begin{array}{c}g_{1}\left(r_{1}\right) \Omega_{1 / 2,1 / 2,0}(1) \\ i f_{1}\left(r_{1}\right) \Omega_{1 / 2,1 / 2,1}^{\prime}(1)\end{array}\right)$,

$|6\rangle=\hat{U}_{c}\left|2 S_{1 / 2},-1 / 2, r_{2}, \zeta\right\rangle=$

$=\left(\begin{array}{c}i f_{1}\left(r_{2}\right) \Omega_{1 / 2,1 / 2,1}^{\prime}(2) \\ g_{1}\left(r_{2}\right) \Omega_{1 / 2,1 / 2,0}(2)\end{array}\right)$.
Here, $r_{1,2}=\sqrt{(z \pm R / 2)^{2}+\rho^{2}}, e^{i \theta_{1,2}}=\frac{z \pm R / 2+i \rho}{r_{1,2}}$, $\rho=\sqrt{x^{2}+y^{2}}$ (see Fig. 4), and the radial functions $f_{i}(r), g_{i}(r), \tilde{f}_{i}(r)$, and $\tilde{g}_{i}(r)$ are determined by numerically integrating the problem with one center (see Section 2).

In addition to the true charge of a nucleus, $\zeta$, two effective charges $\zeta_{a, b}$ are introduced. They are required in order to extend the applicability range of our method onto supercritical nuclear charges. The effective charges have the following values:

$\begin{aligned} \zeta_{a} & = \begin{cases}\zeta, & \zeta<\zeta_{c 1}, \\ 0.99 \zeta_{c 1}, & \zeta \geq \zeta_{c 1},\end{cases} \\ \zeta_{b} & = \begin{cases}\zeta, & \zeta<\zeta_{c 2}, \\ 0.99 \zeta_{c 2}, & \zeta \geq \zeta_{c 2},\end{cases} \end{aligned}$

where $\zeta_{c 1}$ and $\zeta_{c 2}$ are the critical charge values, at which the first and second, respectively, energy levels plunge into the lower continuum.

Let us project the Dirac equation $\hat{H}|\Psi\rangle=E|\Psi\rangle$ onto each of the $|i\rangle$ states $(i=1, \ldots, 6)$ :

$\sum_{j=1}^{6}\left(H_{i j}-E S_{i j}\right) c_{j}=0$.

Then the condition of non-triviality for the coefficients in the linear combination (35) gives rise to the secular equation

$\operatorname{det}\left|H_{i j}-\varepsilon S_{i j}\right|=0$,

where $H_{i j}=\langle i|\hat{H}| j$ are the matrix elements, and $S_{i j}=\langle i \mid j\rangle$ the overlap integrals $(i, j=1, \ldots, 6)$.

The overlap integrals are calculated in Appendix. Of non-diagonal integrals, only 4 are independent; these are $S_{14}, S_{15}, S_{16}$, and $S_{45}$. Therefore, the Gram matrix looks like

$\mathbf{S}=\left(\begin{array}{cccccc}1 & 0 & 0 & S_{14} & S_{15} & S_{16} \\ 0 & 1 & -S_{14} & 0 & -S_{16} & S_{15} \\ 0 & -S_{14} & 1 & 0 & 0 & -S_{45} \\ S_{14} & 0 & 0 & 1 & S_{45} & 0 \\ S_{15} & -S_{16} & 0 & S_{45} & 1 & 0 \\ S_{16} & S_{15} & -S_{45} & 0 & 0 & 1\end{array}\right)$

While calculating the matrix elements, it is convenient to present the total Hamiltonian (20) in the following form:

$\hat{H}(\zeta)=\left\{\begin{array}{l}\hat{H}_{-}\left(\zeta_{i}, r_{1}\right)+\zeta v\left(r_{2}\right)-\left(\zeta-\zeta_{i}\right) v\left(r_{1}\right), \\ \hat{H}_{+}\left(\zeta_{i}, r_{2}\right)-\zeta v\left(r_{1}\right)+\left(\zeta-\zeta_{i}\right) v\left(r_{2}\right),\end{array}\right.$ 


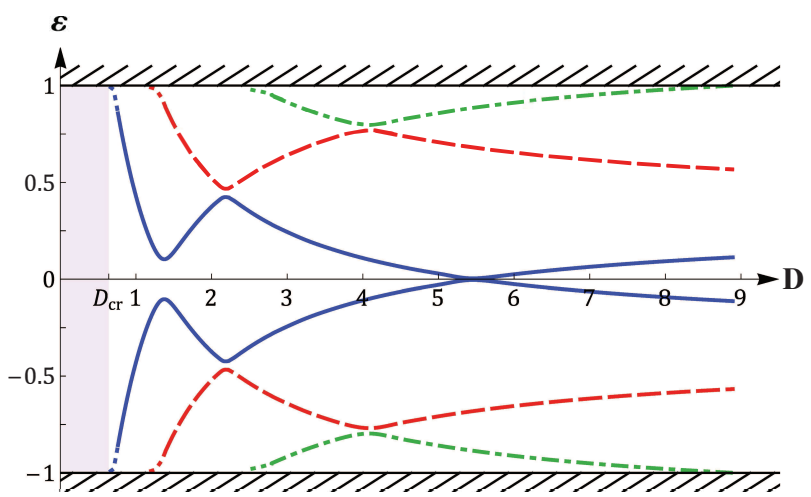

Fig. 7. Dependences of the energy of an electron in the field of an electric dipole on the dimensionless dipole moment $D=$ $\frac{D_{\mathrm{ph}}}{e a_{0}}=\zeta R$ for the nuclear charge $Z=174$. Different types of curves correspond to different energy levels. The tinted area marks the region with no bound states [see formula (2)]

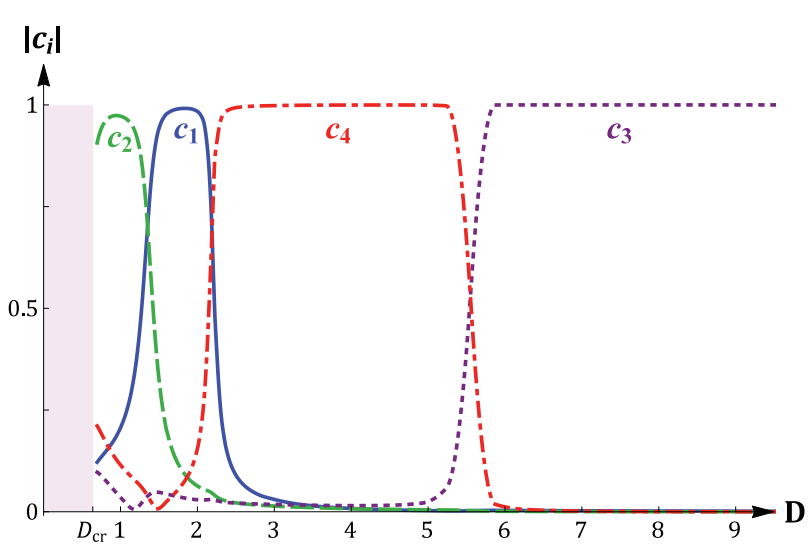

Fig. 8. Dependences of coefficients in the linear combination (35) for the highest filled electron state (the lower solid curve in Fig. 5) on the dimensionless dipole moment $D=\frac{D_{\mathrm{ph}}}{e a_{0}}=\zeta R$ for the nuclear charge $Z=174$. See further explanations in the text

where $\zeta_{i}=\left\{\zeta, \zeta_{a}, \zeta_{b}\right\}$. The advantage of this representation consists in that the states $|i\rangle$ are characteristic of $\hat{H}_{ \pm}$:

$$
\begin{aligned}
& \hat{H}_{-}\left(\zeta_{a}, r_{1}\right)|1\rangle=\varepsilon^{(1 S)}\left(\zeta_{a}\right)|1\rangle, \\
& \hat{H}_{+}\left(\zeta_{a}, r_{2}\right)|2\rangle=-\varepsilon^{(1 S)}\left(\zeta_{a}\right)|2\rangle, \\
& \hat{H}_{-}\left(\zeta_{b}, r_{1}\right)|3\rangle=\varepsilon^{(2 P)}\left(\zeta_{b}\right)|3\rangle, \\
& \hat{H}_{+}\left(\zeta_{b}, r_{2}\right)|4\rangle=-\varepsilon^{(2 P)}\left(\zeta_{b}\right)|4\rangle, \\
& \hat{H}_{-}\left(\zeta, r_{1}\right)|5\rangle=\varepsilon^{(2 S)}(\zeta)|5\rangle, \\
& \hat{H}_{+}\left(\zeta, r_{2}\right)|6\rangle=-\varepsilon^{(2 S)}(\zeta)|6\rangle .
\end{aligned}
$$

The calculation of matrix elements reveals that there are only twelve independent ones:

$$
\begin{aligned}
& H_{11}=-H_{22}=\varepsilon^{(1 S)}\left(\zeta_{a}\right)+\zeta C_{1}^{(2)}-\left(\zeta-\zeta_{a}\right) C_{1}^{(1)}, \\
& H_{12}=-\left(2 \zeta-\zeta_{a}\right) A_{12}^{(1)}, \\
& H_{13}=-H_{24}=\zeta A_{13}^{(2)}, \\
& H_{14}=H_{23}=-\varepsilon^{(2 P)}\left(\zeta_{b}\right) S_{14}-\zeta A_{14}^{(1)}+\left(\zeta-\zeta_{b}\right) A_{14}^{(2)}, \\
& H_{15}=-H_{26}=\varepsilon^{(2 S)}(\zeta) S_{15}+\zeta A_{15}^{(2)}, \\
& H_{16}=H_{25}=-\varepsilon^{(2 S)}(\zeta) S_{16}-\zeta A_{16}^{(1)}, \\
& H_{33}=-H_{44}=\varepsilon^{(2 P)}\left(\zeta_{b}\right)+\zeta C_{3}^{(2)}-\left(\zeta-\zeta_{b}\right) C_{3}^{(1)}, \\
& H_{34}=-\left(2 \zeta-\zeta_{b}\right) A_{34}^{(1)}, \\
& H_{35}=-H_{46}=\zeta A_{35}^{(2)} \\
& H_{36}=H_{45}=\varepsilon^{(2 S)}(\zeta) S_{45}+\zeta A_{45}^{(2)} \\
& H_{55}=-H_{66}=\varepsilon^{(2 S)}(\zeta)+\zeta C_{5}^{(2)} \\
& H_{56}=-\zeta A_{56}^{(1)},
\end{aligned}
$$

where new notations for the exchange, $A_{i j}^{(k)}=$ $=\left\langle i\left|v\left(r_{k}\right)\right| j\right\rangle \quad(i \neq j), \quad$ and $\quad$ Coulomb, $\quad C_{i}^{(k)}=$ $=\left\langle i\left|v\left(r_{k}\right)\right| i\right\rangle$, integrals are introduced. All independent exchange and Coulomb integrals are calculated in Appendix.

The spectrum of the system is found from the secular equation (39), and the coefficients $c_{i}$ of a linear combination are determined as the corresponding normalized characteristic vectors:

$$
(\mathbf{H}-\varepsilon \mathbf{S}) \vec{c}=0,(\vec{c})^{\dagger} \mathbf{S} \vec{c}=1 .
$$

If the nuclei that compose the dipole approach each other very much, they become partially screened and effectively reduce their charges. As they move apart, this mutual screening decreases. Therefore, the growth of the distance between the nuclei is equivalent, to a certain extent, to a gradual increase of the charges at the motionless nuclei from zero to real values.

Figure 7 illustrates the energy dependences for the electron in the field of an electric dipole in the case of nuclear charge $Z=174(\zeta=1.27)$. From Fig. 3, one can see that, at $Z=174$, the first level has already crossed the whole mass gap and plunged

ISSN 2071-0194. Ukr. J. Phys. 2016. Vol. 61, No. 9 
into the lower continuum. At the same time, the second level (for the positive nucleus) has already intersected with the first level for the negative nucleus and also crossed the zero energy value, so that $\varepsilon^{(2 P)}=-0.28<0$. Those facts are reflected in the behavior of the energy and wave functions of the electron in the dipole potential.

The first "constriction" of solid curves in Fig. 7 corresponds to the passage of the first energy level of the electron in the field of one nucleus through the zero value (this case was considered in the previous section). As was shown earlier, the passage of this constriction is accompanied by the migration of the wave function from the negative nucleus to the positive one, which is shown in Fig. 8): the coefficient $c_{2}$ dominates before the constriction, and the coefficient $c_{1}$ after it. Analogously to what was done in the previous section, this migration can be interpreted as the creation of an electron-positron pair from vacuum, with the new particles being in the bound state with the nuclei.

The relative approach and moving away of the solid and dashed curves at $D \approx 2.2$ in Fig. 7 corresponds to the intersection between the first level in the field of the positive nucleus and the second level in the field of the negative nucleus (and vice versa). At this point, an electron transits from the filled second level of the negatively charged nucleus (the dashed curve) to the unfilled first level of the same nucleus (the solid curve). As a result, the coefficient $c_{4}$ starts to dominate (see Fig. 8). Simultaneously, an electron near the positive nucleus transits from the first level to the empty second level, thereby vacating the place and creating preconditions for the next pair to emerge.

Finally, the second constriction in the solid curves in Fig. 7 corresponds to that the second energy level of the electron in the field of one nucleus crosses the zero value. The passage of the constriction is accompanied by the relocalization of the wave function from the negative nucleus to the positive one (the coefficient $c_{4}$ dominates before the constriction, and the coefficient $c_{3}$ after it; see Fig. 8). Similarly to the previous case, this is interpreted as the creation of the second electron-positron pair from vacuum.

Hence, the gradual adiabatic growth of the dipole moment gives rise to the creation of electron-positron pairs from vacuum owing to the phenomenon of wave function migration for the "Dirac sea" electrons. Their maximum number is equal to the number of energy levels of the electron in the field of one nucleus (for a given value of its charge) that crossed the zero energy value.

\section{Conclusions}

To summarize, a new type of supercritical instability of Dirac electrons in the potential of a finite electric dipole composed by two opposite charged nuclei has been studied. In this geometry, the Dirac equation does not allow the separation of variables, so that approximate methods were used. To calculate the spectrum and wave functions of an electron in the field of the dipole, the method of linear combinations of atomic orbitals (LCAO) was applied. This method is rather simple for a realization and allows the majority of calculations to be carried out analytically. At the same time, it is inapplicable at short distances between the impurities. The wave functions of the ground state and several lowest excited states of electron in the potential of one nucleus, which are centered at the corresponding nucleus, were used as atomic orbitals.

If the charge of each nucleus is large enough for the lowest bound state of electron in the field of one of the nuclei to cross the level $E=0$ (i.e. the electron and positron levels should together overlap the interval equal to $2 m c^{2}$ ), the gradual increase of the distance between the center of nuclei results in that the electron and positron levels first start from the corresponding continua and approach, by tending to intersect, each other. However, in accordance with the Wigner-von Neumann theorem concerning the absence of a level crossing, they do not intersect, but start to move apart and asymptotically approach the levels obtained in the field of one center. Thus, the spectrum has a characteristic "constriction". While passing it, the wave function of the electron changes its localization. The electron in the highest filled state migrates from the negatively charged center to the positively charged one. Apparently, it looks as if the electron generated from the Dirac vacuum becomes localized at the positive nucleus and screens it, whereas the positron is localized at the negative nucleus. In other words, similarly to the case of supercritical instability with identically charged nuclei, an electron-positron pair is created; however, now the particles are created in the bound state. It 
is also demonstrated that the further growth of nuclear charges may result in the generation of a larger number of electron-positron pairs.

However, if the nuclear charges are too small for the electron and positron levels, together, to overlap the energy gap $2 m c^{2}$, the described phenomena cannot be observed. Therefore, the supercritical instability in the dipole field has a threshold character.

The threshold values of nuclear charges that are required for the phenomenon of wave-function migration to be observed are $Z \sim 150$. Unfortunately, nuclei with so large charges do not exist in the nature. Moreover, two nuclei with opposite charge signs are needed for this purpose. Those facts make the experimental observation of this phenomenon practically impossible. At the same time, it may hopefully be observed in graphene, because the threshold charge values are comparable in this case with unity, and no problems arise concerning the creation of impurities with opposite charges (positive and negative ions). The impurities can be moved using an electron microscope tip. If the impurities were first put closer to each other and afterward moved a large distance apart, they should be screened. This is a smoking gun of the described phenomenon.

The author expresses his sincere gratitude to E. V. Gorbar and V.P. Gusynin for their valuable advices and corrections made during the discussion of this work. The author also thanks O.I. Voitenko for the qualitative translation of this article from Ukrainian.

\section{APPENDIX}

\section{Overlap, Coulomb, and Exchange Integrals}

In this Appendix, the results of calculations are presented for the overlap, Coulomb, and exchange integrals that arise, when the LCAO method is applied to the problems of an electron in a dipole potential.

Overlap integrals:

$S_{i i}=1, \quad S_{j i}=S_{i j}^{*} \quad i, j=\overline{1,6}$,

$S_{12} \equiv S=\int d^{3} r\left[i g_{0}\left(r_{1}\right) f_{0}\left(r_{2}\right) \Omega^{\dagger}(1) \Omega^{\prime}(2)-\right.$

$\left.-i g_{0}\left(r_{2}\right) f_{0}\left(r_{1}\right) \Omega^{\prime \dagger}(1) \Omega(2)\right]=$

$=\frac{1}{2} \int_{0}^{\infty} \rho d \rho \int_{-\infty}^{\infty} d z\left[g_{0}\left(r_{1}\right) f_{0}\left(r_{2}\right) \cos \theta_{2}+g_{0}\left(r_{2}\right) f_{0}\left(r_{1}\right) \cos \theta_{1}\right]=$

$=\mid$ in the second term $z \rightarrow-z \mid=0$,

$S_{13}=\int d^{3} r\left[i g_{0}\left(r_{1}\right) \tilde{g}_{0}\left(r_{1}\right) \Omega^{\dagger}(1) \Omega^{\prime}(1)+\right.$

$$
\begin{aligned}
& \left.+i f_{0}\left(r_{1}\right) \tilde{f}_{0}\left(r_{1}\right) \Omega^{\prime \dagger}(1) \Omega(1)\right]= \\
& =\frac{1}{4 \pi} \int d^{3} r_{1}\left[g_{0}\left(r_{1}\right) \tilde{g}_{0}\left(r_{1}\right)-f_{0}\left(r_{1}\right) \tilde{f}_{0}\left(r_{1}\right)\right] \cos \theta_{1}=0, \\
& S_{14}=\int d^{3} r\left[g_{0}\left(r_{1}\right) \tilde{f}_{0}\left(r_{2}\right) \Omega^{\dagger}(1) \Omega(2)-\right. \\
& \left.-\tilde{g}_{0}\left(r_{2}\right) f_{0}\left(r_{1}\right) \Omega^{\prime \dagger}(1) \Omega^{\prime}(2)\right]= \\
& =\frac{1}{2} \int_{0}^{\infty} \rho d \rho \int_{-\infty}^{\infty} d z\left[g_{0}\left(r_{1}\right) \tilde{f}_{0}\left(r_{2}\right)-\tilde{g}_{0}\left(r_{2}\right) f_{0}\left(r_{1}\right) \cos \left(\theta_{2}-\theta_{1}\right)\right]= \\
& =\frac{1}{2} \int_{0}^{\infty} \rho d \rho \int_{-\infty}^{\infty} d z\left[g_{0}\left(r_{1}\right) \tilde{f}_{0}\left(r_{2}\right)-\right. \\
& \left.-\frac{z^{2}+\rho^{2}-R^{2} / 4}{r_{1} r_{2}} \tilde{g}_{0}\left(r_{2}\right) f_{0}\left(r_{1}\right)\right], \\
& S_{15}=\int d^{3} r\left[g_{0}\left(r_{1}\right) g_{1}\left(r_{1}\right) \Omega^{\dagger}(1) \Omega(1)+\right. \\
& \left.+f_{0}\left(r_{1}\right) f_{1}\left(r_{1}\right) \Omega^{\prime \dagger}(1) \Omega^{\prime}(1)\right]= \\
& =\int_{0}^{\infty} d r r^{2}\left[g_{0}(r) g_{1}(r)+f_{0}(r) f_{1}(r)\right] .
\end{aligned}
$$

It should be noted that $S_{15}=0$ if $\zeta_{a}=\zeta$, because, in this case, the functions $|1\rangle$ and $|5\rangle$ are characteristic functions of the same Hamiltonian, $\hat{H}_{-}(\zeta)$, but correspond to different energies; therefore, they are orthogonal to each other.

$S_{16}=\int d^{3} r\left[i g_{0}\left(r_{1}\right) f_{1}\left(r_{2}\right) \Omega^{\dagger}(1) \Omega^{\prime}(2)-\right.$

$\left.-i g_{1}\left(r_{2}\right) f_{0}\left(r_{1}\right) \Omega^{\prime \dagger}(1) \Omega(2)\right]=$

$=\frac{1}{2} \int_{0}^{\infty} \rho d \rho \int_{-\infty}^{\infty} d z\left[g_{0}\left(r_{1}\right) f_{1}\left(r_{2}\right) \cos \theta_{2}+\right.$

$\left.+g_{1}\left(r_{2}\right) f_{0}\left(r_{1}\right) \cos \theta_{1}\right]=\mid$ in the second term $z \rightarrow-z \mid=$

$=\frac{1}{2} \int_{0}^{\infty} \rho d \rho \int_{-\infty}^{\infty} d z\left(g_{0}\left(r_{1}\right) f_{1}\left(r_{2}\right)-g_{1}\left(r_{1}\right) f_{0}\left(r_{2}\right)\right) \cos \theta_{2}$,

$S_{23}=\int d^{3} r\left[\tilde{g}_{0}\left(r_{1}\right) f_{0}\left(r_{2}\right) \Omega^{\prime \dagger}(2) \Omega^{\prime}(1)-\right.$

$\left.-g_{0}\left(r_{2}\right) \tilde{f}_{0}\left(r_{1}\right) \Omega^{\dagger}(2) \Omega(1)\right]=$

$=\frac{1}{2} \int_{0}^{\infty} \rho d \rho \int_{-\infty}^{\infty} d z\left[\tilde{g}_{0}\left(r_{1}\right) f_{0}\left(r_{2}\right) \cos \left(\theta_{2}-\theta_{1}\right)-g_{0}\left(r_{2}\right) \tilde{f}_{0}\left(r_{1}\right)\right]=$

$=|z \rightarrow-z|=-\frac{1}{2} \int_{0}^{\infty} \rho d \rho \int_{-\infty}^{\infty} d z\left[g_{0}\left(r_{1}\right) \tilde{f}_{0}\left(r_{2}\right)-\right.$

$\left.-\frac{z^{2}+\rho^{2}-R^{2} / 4}{r_{1} r_{2}} \tilde{g}_{0}\left(r_{2}\right) f_{0}\left(r_{1}\right)\right]=-S_{14}$,

$S_{24}=\int d^{3} r\left[-i g_{0}\left(r_{2}\right) \tilde{g}_{0}\left(r_{2}\right) \Omega^{\dagger}(2) \Omega^{\prime}(2)-\right.$

$\left.-i f_{0}\left(r_{2}\right) \tilde{f}_{0}\left(r_{2}\right) \Omega^{\prime \dagger}(2) \Omega(2)\right]=$

ISSN 2071-0194. Ukr. J. Phys. 2016. Vol. 61, No. 9 
$=-\frac{1}{4 \pi} \int d^{3} r_{2}\left[g_{0}\left(r_{2}\right) \tilde{g}_{0}\left(r_{2}\right)-f_{0}\left(r_{2}\right) \tilde{f}_{0}\left(r_{2}\right)\right] \cos \theta_{2}=0$,

$S_{25}=\int d^{3} r\left[i g_{0}\left(r_{2}\right) f_{1}\left(r_{1}\right) \Omega^{\dagger}(2) \Omega^{\prime}(1)-\right.$

$\left.-i g_{1}\left(r_{1}\right) f_{0}\left(r_{2}\right) \Omega^{\prime \dagger}(2) \Omega(1)\right]=$

$=\frac{1}{2} \int_{0}^{\infty} \rho d \rho \int_{-\infty}^{\infty} d z\left[g_{0}\left(r_{2}\right) f_{1}\left(r_{1}\right) \cos \theta_{1}+\right.$

$\left.+g_{1}\left(r_{1}\right) f_{0}\left(r_{2}\right) \cos \theta_{2}\right]=\mid$ in the first term $z \rightarrow-z \mid=$

$=-\frac{1}{2} \int_{0}^{\infty} \rho d \rho \int_{-\infty}^{\infty} d z\left[g_{0}\left(r_{1}\right) f_{1}\left(r_{2}\right)-\right.$

$\left.-g_{1}\left(r_{1}\right) f_{0}\left(r_{2}\right)\right] \cos \theta_{2}=-S_{16}$,

$S_{26}=\int d^{3} r\left[g_{0}\left(r_{2}\right) g_{1}\left(r_{2}\right) \Omega^{\dagger}(2) \Omega(2)+\right.$

$\left.+f_{0}\left(r_{2}\right) f_{1}\left(r_{2}\right) \Omega^{\prime \dagger}(2) \Omega^{\prime}(2)\right]=$

$=\int_{0}^{\infty} d r r^{2}\left(g_{0}(r) g_{1}(r)+f_{0}(r) f_{1}(r)\right)=S_{15}$.

(A10)

$S_{34}=\int d^{3} r\left[-i \tilde{g}_{0}\left(r_{1}\right) \tilde{f}_{0}\left(r_{2}\right) \Omega^{\prime \dagger}(1) \Omega(2)+\right.$

$\left.+\tilde{g}_{0}\left(r_{2}\right) \tilde{f}_{0}\left(r_{1}\right) \Omega^{\dagger}(1) \Omega^{\prime}(2)\right]=$

$=\frac{1}{2} \int_{0}^{\infty} \rho d \rho \int_{-\infty}^{\infty} d z\left[\tilde{g}_{0}\left(r_{1}\right) \tilde{f}_{0}\left(r_{2}\right) \cos \theta_{1}+\tilde{g}_{0}\left(r_{2}\right) \tilde{f}_{0}\left(r_{1}\right) \cos \theta_{2}\right]=$

$=\mid$ in the second term $z \rightarrow-z \mid=0$,

$S_{35}=\int d^{3} r\left[-i g_{1}\left(r_{1}\right) \tilde{g}_{0}\left(r_{1}\right) \Omega^{\prime \dagger}(1) \Omega(1)-\right.$

$\left.-i f_{1}\left(r_{1}\right) \tilde{f}_{0}\left(r_{1}\right) \Omega^{\dagger}(1) \Omega^{\prime}(1)\right]=$

$=\frac{1}{4 \pi} \int d^{3} r_{1}\left[g_{1}\left(r_{1}\right) \tilde{g}_{0}\left(r_{1}\right)-f_{1}\left(r_{1}\right) \tilde{f}_{0}\left(r_{1}\right)\right] \cos \theta_{1}=0$,

(A12)

$S_{45}=\int d^{3} r\left[g_{1}\left(r_{1}\right) \tilde{f}_{0}\left(r_{2}\right) \Omega^{\dagger}(2) \Omega(1)-\right.$

$\left.-\tilde{g}_{0}\left(r_{2}\right) f_{1}\left(r_{1}\right) \Omega^{\prime \dagger}(2) \Omega^{\prime}(1)\right]=\frac{1}{2} \int_{0}^{\infty} \rho d \rho \int_{-\infty}^{\infty} d z\left[g_{1}\left(r_{1}\right) \tilde{f}_{0}\left(r_{2}\right)-\right.$

$\left.-\frac{z^{2}+\rho^{2}-R^{2} / 4}{r_{1} r_{2}} \tilde{g}_{0}\left(r_{2}\right) f_{1}\left(r_{1}\right)\right]$,

$S_{36}=\int d^{3} r\left[-g_{1}\left(r_{2}\right) \tilde{f}_{0}\left(r_{1}\right) \Omega^{\dagger}(1) \Omega(2)+\right.$

$\left.+\tilde{g}_{0}\left(r_{1}\right) f_{1}\left(r_{2}\right) \Omega^{\prime \dagger}(1) \Omega^{\prime}(2)\right]=$

$=-\frac{1}{2} \int_{0}^{\infty} \rho d \rho \int_{-\infty}^{\infty} d z\left[g_{1}\left(r_{2}\right) \tilde{f}_{0}\left(r_{1}\right)-\right.$

$\left.-\frac{z^{2}+\rho^{2}-R^{2} / 4}{r_{1} r_{2}} \tilde{g}_{0}\left(r_{1}\right) f_{1}\left(r_{2}\right)\right]=|z \rightarrow-z|=-S_{45}$,

$S_{46}=\int d^{3} r\left[i g_{1}\left(r_{2}\right) \tilde{g}_{0}\left(r_{2}\right) \Omega^{\prime \dagger}(2) \Omega(2)+\right.$

$\left.+i f_{1}\left(r_{2}\right) \tilde{f}_{0}\left(r_{2}\right) \Omega^{\dagger}(2) \Omega^{\prime}(2)\right]=$

ISSN 2071-0194. Ukr. J. Phys. 2016. Vol. 61, No. 9

(A9)

(A14)
(A8) $=-\frac{1}{4 \pi} \int d^{3} r_{2}\left[g_{1}\left(r_{2}\right) \tilde{g}_{0}\left(r_{2}\right)-f_{1}\left(r_{2}\right) \tilde{f}_{0}\left(r_{2}\right)\right] \cos \theta_{2}=0$,

$S_{56}=\int d^{3} r\left[i g_{1}\left(r_{1}\right) f_{1}\left(r_{2}\right) \Omega^{\dagger}(1) \Omega^{\prime}(2)-\right.$

$\left.-i g_{1}\left(r_{2}\right) f_{1}\left(r_{1}\right) \Omega^{\prime \dagger}(1) \Omega(2)\right]=$

$=\frac{1}{2} \int_{0}^{\infty} \rho d \rho \int_{-\infty}^{\infty} d z\left[g_{1}\left(r_{1}\right) f_{1}\left(r_{2}\right) \cos \theta_{2}+g_{1}\left(r_{2}\right) f_{1}\left(r_{1}\right) \cos \theta_{1}\right]=$

$=\mid$ in the second term $z \rightarrow-z \mid=0$,

Coulomb integrals:

$C_{1}^{(1)}=\int_{0}^{\infty} d r r^{2}\left(f_{0}^{2}(r)+g_{0}^{2}(r)\right) v(r)$,

$C_{3}^{(1)}=\int_{0}^{\infty} d r r^{2}\left(\tilde{f}_{0}^{2}(r)+\tilde{g}_{0}^{2}(r)\right) v(r)$,

$C_{1}^{(2)} \stackrel{0}{\equiv} C=\int d^{3} r \frac{1}{4 \pi}\left(f_{0}^{2}\left(r_{1}\right)+g_{0}^{2}\left(r_{1}\right)\right) v\left(r_{2}\right)=$

$=\frac{1}{2} \int_{0}^{\infty} r^{2} d r\left(f_{0}^{2}(r)+g_{0}^{2}(r)\right) \times$

$\times \int_{0}^{\pi} d \theta \sin \theta v\left(\sqrt{r^{2}-2 r R \cos \theta+R^{2}}\right)=$

$=\frac{1}{2 R} \int_{0}^{\infty} d r r\left(f_{0}^{2}(r)+g_{0}^{2}(r)\right) \int_{|r-R|}^{r+R} d x x v(x)=$

$=\frac{1}{R} \int_{0}^{R} d r r^{2}\left(f_{0}^{2}(r)+g_{0}^{2}(r)\right)+$

$+\int_{R}^{\infty} d r r\left(f_{0}^{2}(r)+g_{0}^{2}(r)\right)+\mathcal{O}\left(r_{0}^{2}\right)$,

$C_{3}^{(2)}=\frac{1}{R} \int_{0}^{R} d r r^{2}\left(\tilde{f}_{0}^{2}(r)+\tilde{g}_{0}^{2}(r)\right)+$

$+\int_{R}^{\infty} d r r\left(\tilde{f}_{0}^{2}(r)+\tilde{g}_{0}^{2}(r)\right)+\mathcal{O}\left(r_{0}^{2}\right)$,

$C_{5}^{(2)}=\frac{1}{R} \int_{0}^{R} d r r^{2}\left(f_{1}^{2}\left(r_{1}\right)+g_{1}^{2}\left(r_{1}\right)\right)+$

$+\int_{R}^{\infty} d r r\left(f_{1}^{2}\left(r_{1}\right)+g_{1}^{2}\left(r_{1}\right)\right)+\mathcal{O}\left(r_{0}^{2}\right)$.

Exchange integrals:

$A_{12}^{(1)} \equiv A=\frac{1}{2} \int_{0}^{\infty} \rho d \rho \int_{-\infty}^{\infty} d z g_{0}\left(r_{1}\right) \times$

$\times f_{0}\left(r_{2}\right) \frac{z-R / 2}{r_{2}}\left(v\left(r_{1}\right)-v\left(r_{2}\right)\right)$,

$A_{13}^{(2)}=\frac{1}{2} \int_{0}^{\infty} d r r^{2}\left(g_{0}(r) \tilde{g}_{0}(r)-f_{0}(r) \tilde{f}_{0}(r)\right) \times$

$\times \int_{-1}^{1} d x x v\left(\sqrt{r^{2}+R^{2}-2 R r x}\right)$ 


$$
\begin{aligned}
& A_{14}^{(k)}=\frac{1}{2} \int_{0}^{\infty} \rho d \rho \int_{-\infty}^{\infty} d z\left[g_{0}\left(r_{1}\right) \tilde{f}_{0}\left(r_{2}\right)-\right. \\
& \left.-\frac{z^{2}+\rho^{2}-R^{2} / 4}{r_{1} r_{2}} f_{0}\left(r_{1}\right) \tilde{g}_{0}\left(r_{2}\right)\right] v\left(r_{k}\right), k=\overline{1,2}, \\
& A_{15}^{(2)}=\frac{1}{2} \int_{0}^{\infty} d r r^{2}\left(g_{0}(r) g_{1}(r)+f_{0}(r) f_{1}(r)\right) \times \\
& \times \int_{-1}^{1} d x v\left(\sqrt{r^{2}+R^{2}-2 R r x}\right)= \\
& =\frac{1}{R} \int_{0}^{R} d r r^{2}\left(g_{0}(r) g_{1}(r)+f_{0}(r) f_{1}(r)\right)+\tilde{f}_{0}\left(r_{2}\right)+ \\
& +\int_{R}^{\infty} d r r\left(g_{0}(r) g_{1}(r)+f_{0}(r) f_{1}(r)\right)+\mathcal{O}\left(r_{0}^{2}\right), \\
& A_{16}^{(1)}=\frac{1}{2} \int_{0}^{\infty} \rho d \rho \int_{-\infty}^{\infty} d z\left(g_{0}\left(r_{1}\right) f_{1}\left(r_{2}\right)-\right. \\
& \left.-f_{0}\left(r_{2}\right) g_{1}\left(r_{1}\right)\right) \cos \theta_{2} v\left(r_{1}\right), \\
& A_{34}^{(1)}=\frac{1}{2} \int_{0}^{\infty} \rho d \rho \int_{-\infty}^{\infty} d z \tilde{g}_{0}\left(r_{1}\right) \times \\
& \times \tilde{f}_{0}\left(r_{2}\right) \frac{z+R / 2}{r_{1}}\left(v\left(r_{1}\right)-v\left(r_{2}\right)\right), \\
& A_{35}^{(2)}=\frac{1}{2} \int_{0}^{\infty} d r r^{2}\left(g_{1}(r) \tilde{g}_{0}(r)-f_{1}(r) \tilde{f}_{0}(r)\right) \times \\
& \times \int_{-1}^{1} d x x v\left(\sqrt{r^{2}+R^{2}-2 R r x}\right) \\
& A_{45}^{(2)}=\frac{1}{2} \int_{0}^{\infty} \rho d \rho \int_{-\infty}^{\infty} d z\left[g_{1}\left(r_{1}\right) \tilde{f}_{0}\left(r_{2}\right)-\right. \\
& \left.-\frac{z^{2}+\rho^{2}-R^{2} / 4}{r_{1} r_{2}} f_{1}\left(r_{1}\right) \tilde{g}_{0}\left(r_{2}\right)\right] v\left(r_{2}\right), \\
& A_{56}^{(1)}=\frac{1}{2} \int_{0}^{\infty} \rho d \rho \int_{-\infty}^{\infty} d z g_{1}\left(r_{1}\right) \times \\
& \times f_{1}\left(r_{2}\right) \frac{z-R / 2}{r_{2}}\left(v\left(r_{1}\right)-v\left(r_{2}\right)\right) .
\end{aligned}
$$

1. P.A.M. Dirac, The quantum theory of the electron. Part II, Proc. R. Soc. London, A 118, 351 (1928) [DOI: 10.1098/rspa.1928.0056].

2. C.G. Darwin, The wave equations of the electron, Proc. R. Soc. London, A 118, 654 (1928) [DOI: 10.1098/rspa.1928.0076].

3. W. Gordon, Die energieniveaus des wasserstoffatoms nach der diracschen quantentheorie des elektrons, Z. Phys. 48, 11 (1928) [DOI: 10.1007/BF01351570].
4. I.Ya. Pomeranchuk and Y.A. Smorodinsky, On energy levels in systems with $Z>137, J$. Phys. USSR 9, 97 (1945).

5. Ya.B. Zeldovich and V.N. Popov, Electronic structure of superheavy atoms, Sov. Phys. Usp. 14, 673 (1972) [DOI: 10.1070/PU1972v014n06ABEH004735].

6. W. Greiner, B. Müller, and J. Rafelski, Quantum Electrodynamics of Strong Fields (Springer, Berlin, 1985).

7. S.S. Gershtein and Ya.B. Zeldovich, Positron production during the mutual approach of heavy nuclei and the polarization of the vacuum, Sov. Phys. JETP 30, 358 (1970).

8. J. Rafelski, L.P. Fulcher, and W. Greiner, Superheavy elements and an upper limit to the electric field strength, Phys. Rev. Lett. 27, 958 (1971).

9. B. Müller, H. Peitz, J. Rafelski, and W. Greiner, Solution of the Dirac equation for strong external fields, Phys. Rev. Lett. 28, 1235 (1972) [DOI: 10.1103/PhysRevLett.28.1235].

10. M.S. Marinov and V.S. Popov, Critical distance in collision of heavy ions, Sov. Phys. JETP 41, 205 (1975).

11. K.S. Novoselov, A.K. Geim, S.V. Morozov et al., Electric field effect in atomically thin carbon films, Science 306, 666 (2004) [DOI: 10.1126/science.1102896].

12. V.P. Gusynin, S.G. Sharapov, and J.P. Carbotte, $\mathrm{AC}$ conductivity of graphene: from tight-binding model to $2+1$-dimensional quantum electrodynamics, Int. J. Mod. Phys. B 21, 4661 (2007) [DOI: 10.1142/S0217979207038022].

13. A.H. Castro Neto, F. Guinea, N.M.R. Peres, K.S. Novoselov, and A.K. Geim, The electronic properties of graphene, Rev. Mod. Phys. 81, 109 (2009) [DOI: 10.1103/RevModPhys.81.109].

14. D.S.L. Abergel, V. Apalkov, J. Berashevich, K. Ziegler, and T. Chakraborty, Properties of graphene: A theoretical perspective, Adv. Phys. 59, 261 (2010) [DOI: 10.1080/00018732.2010.487978].

15. G. Giovanetti, P.A. Khomyakov et al., Substrate-induced band gap in graphene on hexagonal boron nitride: $A b$ initio density functional calculations, Phys. Rev. B 76, 073103 (2007) [DOI: 10.1103/PhysRevB.76.073103].

16. L.A. Ponomarenko et al., Nature 497, 594 (2013).

17. O.V. Gamayun, E.V. Gorbar, and V.P. Gusynin, Supercritical Coulomb center and excitonic instability in graphene, Phys. Rev. B 80, 165429 (2009) [DOI: 10.1103/PhysRevB.80.165429].

18. Y. Wang et al., Observing atomic collapse resonances in artificial nuclei on graphene, Science 340, 734 (2013) [DOI: 10.1126/science.1234320].

19. O.O. Sobol, E.V. Gorbar, and V.P. Gusynin, Supercritical instability in graphene with two charged impurities, Phys. Rev. B 88, 205116 (2013) [DOI: 10.1103/PhysRevB.88.205116].

20. O.O. Sobol, Variational method for the calculation of critical distance between two Coulomb centers in graphene, Ukr. J. Phys. 59, 531 (2014).

ISSN 2071-0194. Ukr. J. Phys. 2016. Vol. 61, No. 9 
21. A. de Martino, D. Klopfer, D. Matrasulov, and R. Egger, Electric-dipole-induced universality for Dirac fermions in graphene Phys. Rev. Lett. 112, 186603 (2014) [DOI: 10.1103/PhysRevLett.112.186603].

22. E.V. Gorbar, V.P. Gusynin, and O.O. Sobol, Supercritical electric dipole and migration of electron wave function in graphene, Europhys. Lett. 111, 37003 (2015) [DOI: 10.1209/0295-5075/111/37003].

23. E.V. Gorbar, V.P. Gusynin, and O.O. Sobol, Supercriticality of novel type induced by electric dipole in gapped graphene, Phys. Rev. B 92, 235417 (2015) [DOI: 10.1103/PhysRevB.92.235417].

24. E. Fermi and E. Teller, The capture of negative mesotrons in matter, Phys. Rev. 72, 399 (1947) [DOI: 10.1103/PhysRev.72.399].

25. J.E. Turner, Minimum dipole moment required to bind an electron-molecular theorists rediscover phenomenon mentioned in Fermi-Teller paper twenty years earlier, Am. J. Phys. 45, 758 (1977) [DOI: 10.1119/1.10767].

26. D.I. Abramov and V.I. Komarov, Weakly bound states of a charged particle in a finite-dipole field, Theor. Math. Phys. 13, 1090 (1972) [DOI: 10.1007/BF01035530].

27. K. Connolly and D.J. Griffiths, Critical dipoles in one, two, and three dimensions, Am. J. Phys. 75, 524 (2007) [DOI: 10.1119/1.2710485].

28. V.I. Matveev, M.M. Musakhanov, and D.U. Matrasulov, Dirac electron in the electric dipole field, hep-th/9501027.

29. J. von Neumann and E.P. Wigner, Über die analytischen eigenschaften von gruppen linearer transformationen und ihrer darstellungen, Z. Phys. 30, 467 (1929) [DOI: 10.1007/BF01187749].
30. L.D. Landau and E.M. Lifshitz, Quantum Mechanics. Non-Relativistic Theory (Pergamon Press, New York, 1977).

31. H.A. Bethe and E.E. Salpeter, Quantum Mechanics of One- and Two-Electron Atoms (Springer, Berlin, 1957).

32. P. Marmier and E. Sheldon, Physics of Nuclei and Particles, Vol. 1 (Academic Press, New York, 1969).

33. C. Cohen-Tannoudji, B. Diu, and F. Laloe, Quantum Mechanics, Vol. 2 (Hermann, Paris, 1977).

Received 14.09.15.

Translated from Ukrainian by O.I. Voitenko

\section{О.О. Соболь}

\section{НАДКРИТИЧНА НЕСТАБІЛЬНІСТЬ}

ДІРАКІВСЬКИХ ЕЛЕКТРОНІВ У ПОЛІ ДВОХ

ПРОТИЛЕЖНО ЗАРЯДЖЕНИХ ЯДЕР

Р е $з$ ю м е

$\mathrm{Y}$ роботі досліджено рівняння Дірака для електрона в потенціалі скінченного електричного диполя за допомогою техніки лінійних комбінацій атомних орбіталей (ЛКАО). Кулонівський потенціал ядер, що утворюють диполь, регуляризований шляхом врахування їх скінченних розмірів. Показано, що при перевищенні деякого критичного значення дипольного моменту спостерігається новий тип надкритичної нестабільності: хвильова функція найвищого заповненого електронного рівня змінює свою локалізацію з від'ємно зарядженого ядра на додатно заряджене, що можна проінтерпретувати як спонтанне народження з вакууму пари електрона і позитрона, кожен із яких знаходиться у зв'язаному стані з відповідним ядром і частково його екранує. 